\title{
INSTRUMENTOS PARA EVALUAR Y CALIFICAR DE FORMA DEMOCRÁTICA Y COMPRENSIBLE EN EDUCACIÓN PRIMARIA
}

Instruments for democratic assesment in the Elementary School.

Instrumentos de avaliação e qualificação democrática e comprensible no ensino primário

\section{Pablo Casado Berrocal}

CEIP “Villa y Tierra” de Saldaña (Palencia), España. Teléfono +34 660821826. Correo electrónico: pablocasado5@gmail.com

\section{Resumen}

La experiencia presenta los resultados obtenidos en el proceso inicial de renovación pedagógica y de evaluación de una escuela rural. Durante un curso académico se utilizaron técnicas y recursos relacionados con la Escuela Moderna y con los procesos de evaluación formativa y compartida en dos grupos de sexto de Educación Primaria. La observación sistemática y las entrevistas realizadas a los alumnos durante estos procesos permitieron identificar un problema: los alumnos no entendían el proceso de evaluación. Una de las razones más habituales de este problema es que los docentes solemos emplear instrumentos de evaluación con un lenguaje que el alumno no logra comprender parcialmente. Es, por tanto, necesario dotar a los alumnos de instrumentos que permitan traducir "a su lenguaje” los logros, dificultades y decisiones tomadas a partir del proceso de evaluación. Un ejemplo de "instrumento traductor" es la escalera de color que se presenta en el texto.

Palabras clave: Escuela Moderna; evaluación formativa y compartida; instrumentos de evaluación

\begin{abstract}
The experience presents the results obtained in the process of pedagogical renewal and evaluation put into practice in a rural school. During an academic year, techniques and resources related to the Modern School and to the processes of formative and shared evaluation were used. In these processes, a problem was identified: students do not understand their own evaluation process. One of the most common reasons for this problem is that teachers use evaluation tools with a language that the student fails to Instrumentos para evaluar y calificar de forma democrática y comprensible en educación primaria
\end{abstract}


understand. It is therefore necessary to provide students with tools that enable them to translate their achievements, difficulties and decisions made through the evaluation process into their language in a practical, quick and visual way. An example of "translator instrument" is the color ladder that is presented throughout this text.

Keywords: Modern School; formative and shared assessment; assessment instruments

\section{Resumo}

Experiência apresenta os resultados obtidos no processo de reforma do ensino e avaliação implementado em uma escola rural. Durante um ano, e técnicas relacionadas com a Escola Moderna e processos formativos e avaliação de recursos compartilhados acadêmica eles foram usados. Nestes processos, um problema foi identificado: os alunos não entendem o seu próprio processo de avaliação. Uma das razões mais comuns para este problema é que as ferramentas de avaliação de professores usamos a linguagem que o aluno não consegue entender. Portanto, é necessário dotar os alunos de instrumentos para traduzir "o seu idioma" práticos, rápidos e visuais, realizações, dificuldades e decisões do processo de avaliação. Um exemplo de "tradutor instrumento" é o straight flush apresentadas ao longo deste texto.

Palavras-chave: Escola Moderna; avaliação formativa e compartilhada; ferramentas de avaliação

\section{Introducción}

Desde la perspectiva de una Escuela Moderna (Freinet, 2005), uno de los aspectos que genera mayor preocupación y debate es la evaluación y los procesos asociados a ella.

Desde hace algunos años son frecuentes las situaciones en las que el alumno reflexiona y dialoga con el docente acerca de su evaluación y de sus resultados o calificaciones (Pérez-Pueyo, 2013; Santos-Guerra, 2012). Es en este proceso de diálogo en el que se suele identificar un problema bastante extendido: a veces los alumnos toman la palabra en su propio proceso de evaluación sin entender los criterios con los que este se ha llevado a cabo.

En muchas ocasiones, el problema tiene raíz en la siguiente afirmación: los docentes empleamos instrumentos de evaluación/calificación con un lenguaje que el 
alumnado no logra entender. En Educación Primaria, por ejemplo, es común escuchar a docentes explicar el proceso de evaluación y de calificación a sus alumnos en términos de porcentajes, números decimales o reglas de tres cuando todavía no tienen noción de esos conceptos.

Es, por tanto, relevante, dotar a los alumnos de instrumentos que permitan traducir "a su lenguaje” de forma rápida y visual, los logros, dificultades y decisiones tomadas a partir del proceso de evaluación, para poder reconducir con éxito su propio aprendizaje. Un ejemplo de "instrumento traductor" es la escalera de color (CasadoBerrocal, 2017) que se presenta a lo largo de este texto.

\section{Contextualización}

La experiencia muestra los resultados obtenidos al emplear, entre otros, la escalera de color como instrumento de evaluación traductor en el área de Ciencias de la Naturaleza en un CEIP rural de doble línea que cuenta con 436 alumnos. En ella, han participado 45 alumnos de $6^{\circ}$ de Educación Primaria divididos en dos grupos (A y B) de 23 y 22 alumnos respectivamente.

Junto a los procesos de evaluación formativa y compartida (Pérez-Pueyo, 2013) se pusieron en práctica técnicas y estrategias vinculadas al Aprendizaje Cooperativo (Zariquiey, 2015), a la Autorregulación (Zimmerman \& Schunk, 1990) y al Aprendizaje Basado en Proyectos (Majó \& Baqueró, 2014).

\section{Diseño}

La fase de diseño de la experiencia se desarrolla durante el primer trimestre del curso académico, llevándose a cabo, entre otros, los siguientes procesos:

(1) Sondeo realizado a cada grupo de alumnos sobre la forma en la que eran evaluados y calificados en el área en cursos anteriores (información y valoración empleando cuestionarios con preguntas abiertas y cerradas).

(2) Selección de procesos, actividades y momentos susceptibles de evaluación y de calificación elegidos en dos reuniones del Equipo de Nivel de $6^{\circ}$.

(3) Renovación: Diseño de nuevos instrumentos de evaluación y/o calificación para cada momento/categoría y sus criterios de calificación. 
(4) Consolidación de un único instrumento traductor de evaluación y calificación para el alumno: la escalera de color (instrumento que se detalla más adelante).

\section{Desarrollo}

La fase de desarrollo de la experiencia comprende los meses de octubre a junio, y en ella se introdujo de forma sistemática el uso de la escalera de color como instrumento traductor.

La última versión empleada por los alumnos fue la siguiente (Figura 1).

\begin{tabular}{|c|c|}
\hline \multirow{3}{*}{$\begin{array}{l}\text { Cuaderno y } \\
\text { fichas }\end{array}$} & Presentación: Cumplo las normas y CORRIJO todas las actividades. \\
\hline & Cuidado de materiales: Mi cuaderno y fichas parecen casi nuevos. \\
\hline & Contenido: Comprendo lo que me piden y contesto bien casi siempre. \\
\hline \multirow{3}{*}{$\begin{array}{l}\text { Proyecto y } \\
\text { talleres }\end{array}$} & Colaboración: He colaborado con mis compañeros al trabajar. \\
\hline & Cuidado: He cuidado los materiales y no ha habido accidentes. \\
\hline & Conocimiento: He resuelto la actividad correctamente sin mucha ayuda. \\
\hline \multirow{3}{*}{$\begin{array}{l}\text { Trabajo en } \\
\text { clase }\end{array}$} & Atención: Sigo la explicación y entiendo las instrucciones que me dan. \\
\hline & Comportamiento: Me porto bien. Muestro educación y respeto. \\
\hline & Planificación: He cumplido mi plan de trabajo desde el principio. \\
\hline \multirow{3}{*}{$\begin{array}{l}\text { Preguntas } \\
\text { orales u } \\
\text { escritas }\end{array}$} & $\begin{array}{l}\text { Conocimiento y atención: Contesto bien prácticamente siempre a las } \\
\text { preguntas de Plickers. }\end{array}$ \\
\hline & Participación: Me muestro voluntario en los "rebotes". \\
\hline & $\begin{array}{l}\text { Conocimiento y atención: Respondo correctamente a las preguntas } \\
\text { escritas que me hacen sobre el tema o sobre las exposiciones. }\end{array}$ \\
\hline \multirow{4}{*}{ Exposición } & $\begin{array}{l}\text { Colaboración: 1. Hay buena organización, sin despistes, y cada } \\
\text { componente participa prácticamente el mismo tiempo. } 2 \text {. Antes de } \\
\text { comenzar, se presentan. } 3 \text {. La exposición es original y entretenida. } 4 \text { y } 5 \text {. } \\
\text { Los materiales (PowerPoint, cartulina, etc.) tienen calidad y denotan } \\
\text { esfuerzo. 6. Al terminar preguntan al público por si hubiese alguna duda. }\end{array}$ \\
\hline & $\begin{array}{l}\text { Comportamiento: } 1 \text {. Muestro buena actitud y respeto cuando exponen } \\
\text { mis compañeros. } 2 \text {. Me muestro responsable con mi tarea. }\end{array}$ \\
\hline & $\begin{array}{l}\text { Participación: } 1 \text {. Me muestro tranquilo, sin dudar y sin "atascarme". } \\
\text { 2. Ocupo el espacio. } 3 \text {. Me dirijo a todo el público sin mirar diapositivas. } \\
\text { 4. Lo que explico se entiende y consigo "enganchar" al público. }\end{array}$ \\
\hline & Conocimiento: 1,2 y 3 . El contenido que explico es correcto. \\
\hline
\end{tabular}

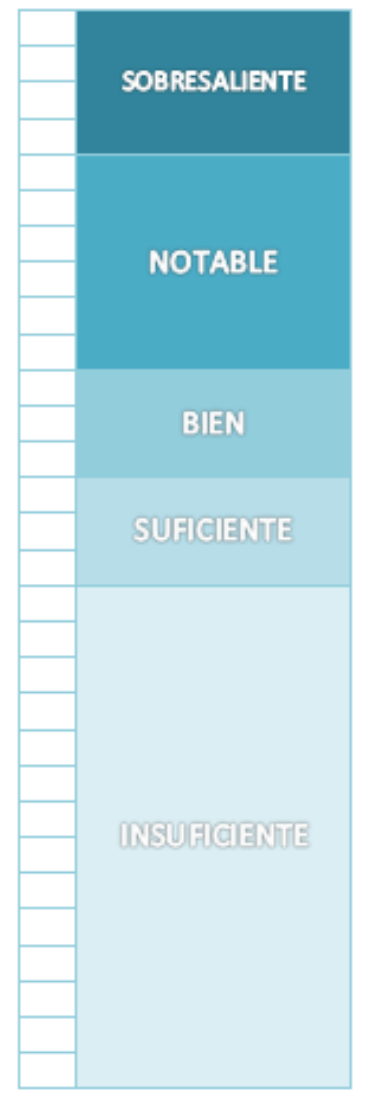

Figura 1.

Última versión de la escalera de color empleada por los alumnos de $6^{\circ}$ de Educación Primaria en Ciencias de la Naturaleza (elaboración propia).

Con la escalera de color, el alumno rápidamente puede entender:

(1) Categorías susceptibles de calificación, las que se indican en la primera columna de la izquierda.

(2) Peso de cada categoría en la calificación, ya que la altura de cada categoría es proporcional a su peso en la calificación. Por ejemplo, como la categoría “Exposición” ocupa 15 cuadritos, equivale al 50\% de la calificación, mientras 
que la categoría “Cuadernos y fichas”, por su tamaño, tan solo equivale al 10\% de la calificación.

(3) Aspectos de su aprendizaje (cuidado de materiales, atención, comportamiento, conocimiento, etc.) y evidencia/actividad con la que se valora (cuaderno, talleres, etc.)

(4) Calificación total de la unidad/tema, ya que el alumno determina qué aspectos/cuadritos ha cumplido y los va coloreando en su escalera de color hasta llegar al nivel que corresponde (por ejemplo, notable, sobresaliente, etc.)

(5) Aspectos no logrados o mejorables, ya que los cuadraditos no coloreados, aún no están conseguidos y carecen de valoración positiva.

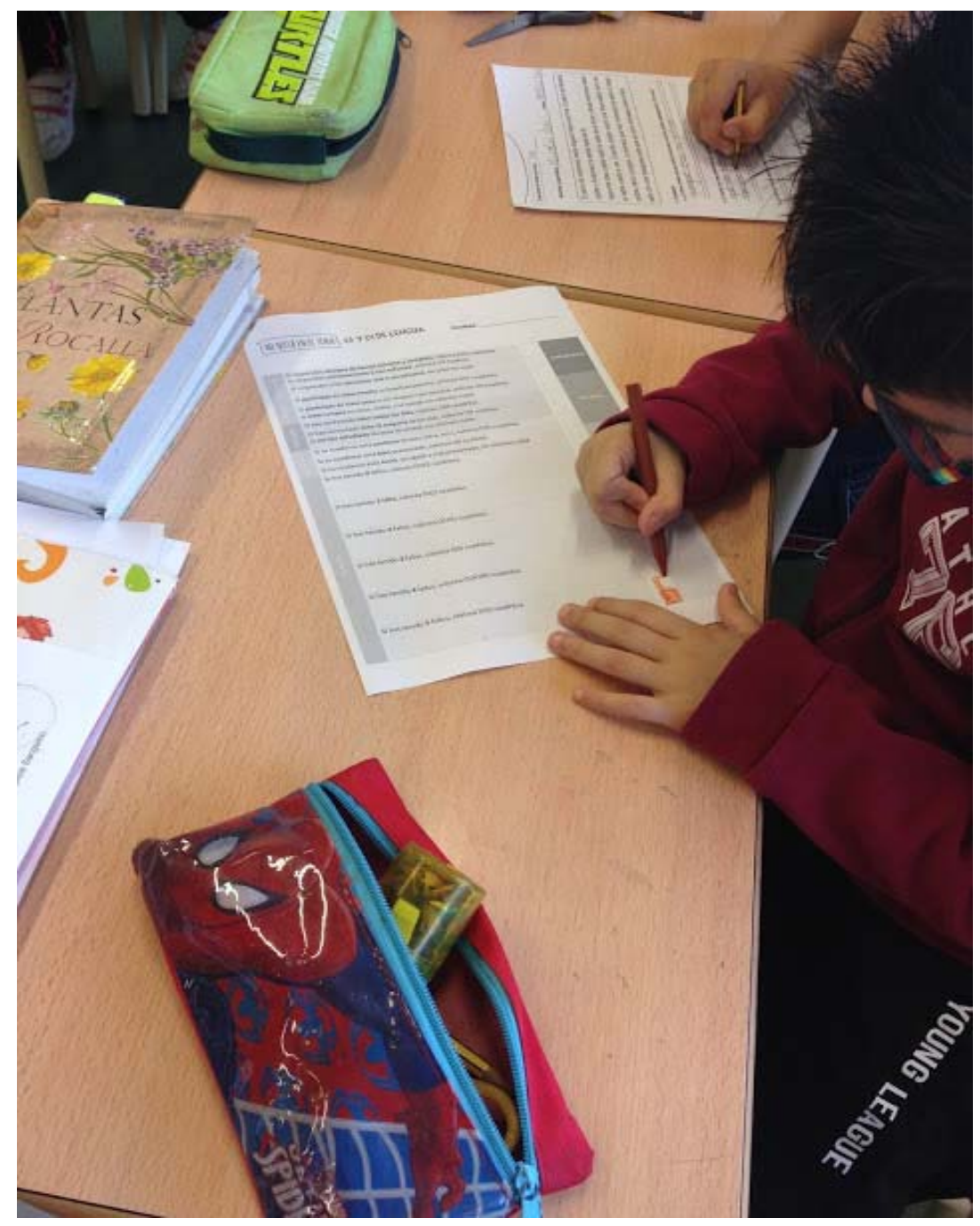

Figura 2.

Alumno de $2^{\circ}$ de Educación Primaria coloreando los cuadritos conseguidos para conocer la calificación obtenida. 
Esos cuadritos no coloreados indicarían aquello que el alumno puede mejorar. Sin embargo, con la intención de llevar a cabo un proceso coherente de evaluación, no solo se indica qué puede mejorar sino la forma en la que puede mejorar esos aspectos. Por ello, junto a la escalera de color (Figura 3) se recoge la siguiente tabla en la que se ofrece al alumno un abanico de propuestas para mejorar los aspectos necesarios de su evaluación (Figura 4).

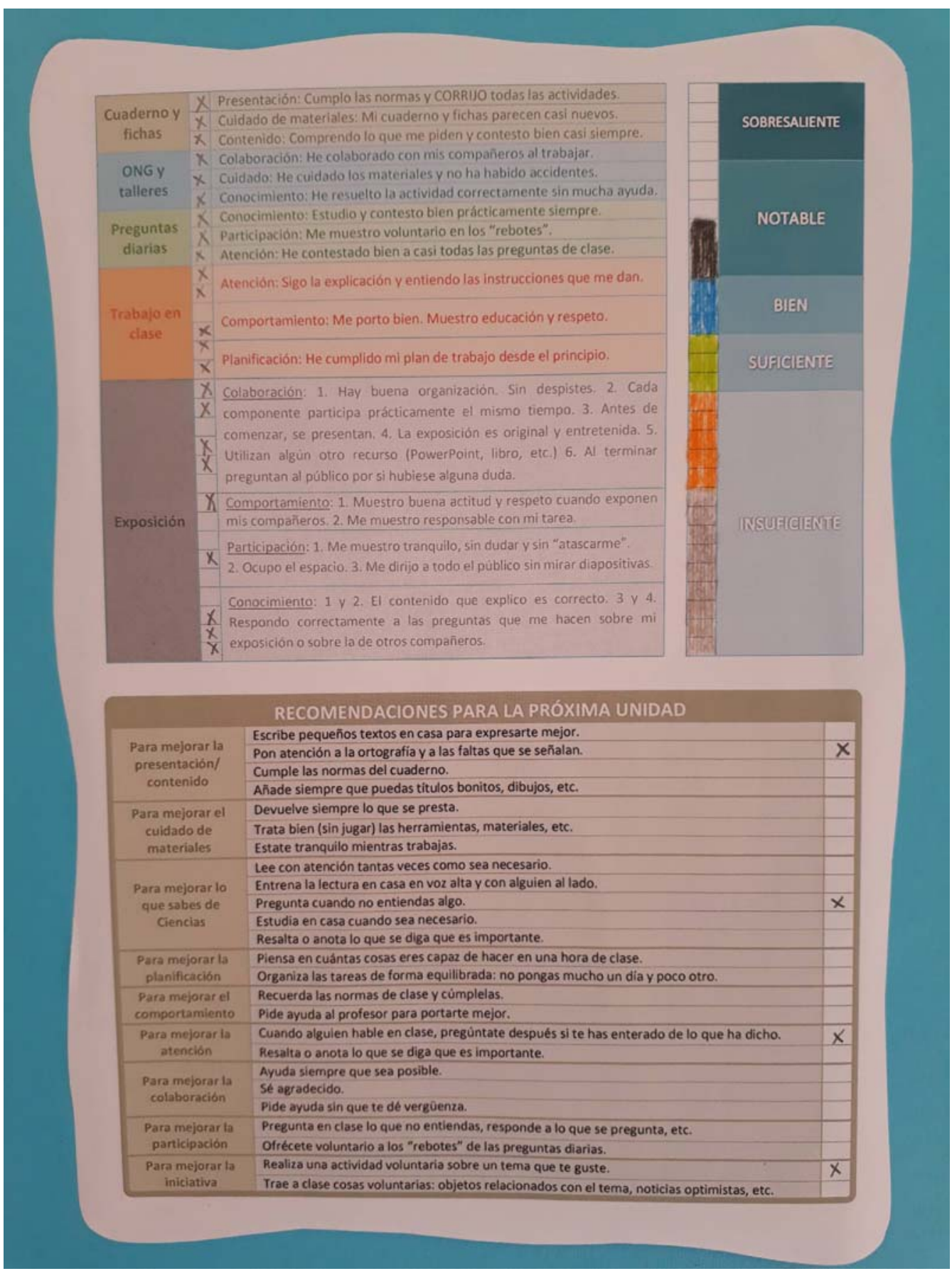

Figura 3.

Escalera de color de un alumno de $6^{\circ}$ tras su utilización. 


\begin{tabular}{|c|c|c|c|}
\hline \multicolumn{4}{|c|}{ RECOMENDACIONES PARA LA PRÓXIMA UNIDAD } \\
\hline \multirow{4}{*}{\multicolumn{2}{|c|}{$\begin{array}{l}\text { Para mejorar la } \\
\text { presentación/ } \\
\text { contenido }\end{array}$}} & & Escribe pequeños textos en casa para expresarte mejor. \\
\hline & & & Pon atención a la ortografía y a las faltas que se señalan. \\
\hline & & & Cumple las normas del cuaderno. \\
\hline & & & Añade siempre que puedas títulos bonitos, dibujos, etc. \\
\hline \multirow{3}{*}{\multicolumn{2}{|c|}{$\begin{array}{l}\text { Para mejorar el } \\
\text { cuidado de } \\
\text { materiales }\end{array}$}} & & Devuelve siempre lo que se presta. \\
\hline & & & Trata bien (sin jugar) las herramientas, materiales, etc. \\
\hline & & & Estate tranquilo mientras trabajas. \\
\hline \multirow{5}{*}{\multicolumn{2}{|c|}{$\begin{array}{c}\text { Para mejorar lo } \\
\text { que sabes de } \\
\text { Ciencias }\end{array}$}} & & Lee con atención tantas veces como sea necesario. \\
\hline & & & Entrena la lectura en casa en voz alta y con alguien al lado. \\
\hline & & & Pregunta cuando no entiendas algo. \\
\hline & & & Estudia en casa cuando sea necesario. \\
\hline & & & Resalta o anota lo que se diga que es importante. \\
\hline \multirow{2}{*}{\multicolumn{2}{|c|}{$\begin{array}{c}\text { Para mejorar la } \\
\text { planificación }\end{array}$}} & & Piensa en cuántas cosas eres capaz de hacer en una hora de clase. \\
\hline & & & Organiza las tareas de forma equilibrada: no pongas mucho un día y poco otro. \\
\hline \multirow{2}{*}{\multicolumn{2}{|c|}{$\begin{array}{l}\text { Para mejorar el } \\
\text { comportamiento }\end{array}$}} & & Recuerda las normas de clase y cúmplelas. \\
\hline & & & Pide ayuda al profesor para portarte mejor. \\
\hline \multirow{2}{*}{\multicolumn{2}{|c|}{$\begin{array}{c}\text { Para mejorar la } \\
\text { atención }\end{array}$}} & & Cuando alguien hable en clase, pregúntate después si te has enterado de lo que ha dicho. \\
\hline & & & Resalta o anota lo que se diga que es importante. \\
\hline \multirow{3}{*}{\multicolumn{2}{|c|}{$\begin{array}{c}\text { Para mejorar la } \\
\text { colaboración }\end{array}$}} & & Ayuda siempre que sea posible. \\
\hline & & & Sé agradecido. \\
\hline & & & Pide ayuda sin que te dé vergüenza. \\
\hline \multirow{2}{*}{\multicolumn{2}{|c|}{$\begin{array}{c}\text { Para mejorar la } \\
\text { participación }\end{array}$}} & & Pregunta en clase lo que no entiendas, responde a lo que se pregunta, etc. \\
\hline & & & Ofrécete voluntario a los "rebotes" de las preguntas diarias. \\
\hline \multirow{2}{*}{\multicolumn{2}{|c|}{$\begin{array}{l}\text { Para mejorar la } \\
\text { iniciativa }\end{array}$}} & & Realiza una actividad voluntaria sobre un tema que te guste. \\
\hline & & & Trae a clase cosas voluntarias: objetos relacionados con el tema, noticias optimistas, etc. \\
\hline
\end{tabular}

Figura 4.

Recomendaciones complementarias a la escalera de color.

\section{Evaluación y conclusiones}

La experiencia recogida en el texto arroja resultados positivos en diversas categorías que dejan abierto el camino para una futura línea de investigación que cuente con datos estadísticos fiables y más objetivos. Por el momento, se pone de manifiesto que:

- La utilización de la escalera de color facilitó que cada alumno fuera capaz de justificar la razón de sus resultados.

- Las recomendaciones complementarias a la escalera de color permitieron una mejora generalizada de los resultados de evaluación.

- El diseño de momentos variados de evaluación con intención formativa permitió una mejora visible en las destrezas de expresión escrita, expresión oral y trabajo en equipo.

- La comprensión del proceso de evaluación/calificación resultó prácticamente plena por parte de los familiares del grupo. 
Estas observaciones denotan la importancia que tuvo hacer comprensibles los procesos de evaluación y de calificación con los dos grupos de $6^{\circ}$ de Educación Primaria.

Con la información recogida en este documento se ha intentado describir una experiencia que sirva para animar a otros docentes a diseñar instrumentos traductores en cualquier etapa educativa, con la intención de conseguir procesos de evaluación coherentes y comprensibles por el alumnado, su familia y cualquier otra persona implicada en el proceso educativo.

\section{Referencias}

Freinet, C. (2005). Técnicas Freinet para la Escuela Moderna. Madrid: Siglo XXI.

Freinet, C., \& Salengros, R. (1976). Modernizar la escuela. Barcelona: Laia.

Johnson, D., \& Johnson, R. (1999). El aprendizaje cooperativo en el aula. Buenos Aires: Paidós.

Majó, F., \& Baqueró, M. (2014). Los proyectos interdisciplinarios. Ocho ideas clave. Barcelona: Graó.

Pérez-Pueyo, Á. (2014). La Evaluación Formativa y Compartida en el Marco del Estilo Actitudinal. Des-encuentros, 10, 6-17.

Pujolás, P. (2011). El aprendizaje cooperativo. Nueve ideas clave. Barcelona: Graó.

Santos-Guerra, M.Á. (2012). La pedagogía contra Frankenstein. Barcelona: Graó.

Zariquiey, F. (2015). Guía para diseñar y poner en marcha una red de aprendizaje cooperativo. Madrid: Colectivo Cinética.

Zimmerman, B.J., \& Schunk, D.H. (1990). Self-regulated learning and academic achievement. Educational Psychologist, 25(1), 3-17. 